\title{
THE ASYMPTOTIC BEHAVIOR OF A FOURIER TRANSFORM AND THE LOCALIZATION PROPERTY FOR EIGENFUNCTION EXPANSIONS FOR SOME PARTIAL DIFFERENTIAL OPERATORS
}

\author{
BY \\ BURTON RANDOL
}

\begin{abstract}
The asymptotic behavior of a certain Fourier transform is investigated, and the result is applied to obtain a localization theorem for elliptic operators on the torus.
\end{abstract}

Let $T(x)$ be a positive analytic function, homogeneous of weight 1 , in $R^{n}-\{0\}$ $(n>1)$. Define $C=\{x \mid T(x) \leqq 1\}, \partial C=\{x \mid T(x)=1\}$. This note is about the asymptotic behavior, as $r \rightarrow \infty$, of the Fourier transform

$$
F_{\delta}(r, \theta)=\int_{C}\left(1-(T(x))^{2}\right)^{\delta} e^{i r(\theta, x)} d v_{x},
$$

where $0<\delta \leqq[(n-1) / 2]+1, \theta \in S^{n-1}, r=|x|$, and $d v_{x}=$ the volume element in $R^{n}$. In case $\partial C$ has everywhere positive Gaussian curvature, the asymptotic behavior of $F_{\delta}(r, \theta)$ has been discussed by Hlawka [5].

Such integrals arise in the extension of the localization theorems for summation of Fourier series by Riesz means to certain types of eigenfunction expansions for more general elliptic operators. That estimates of the type contained here would be useful in this context was pointed out to me by E. M. Stein, and the present note is a result of that conversation.

In order to estimate $F_{\delta}(r, \theta)$, it is necessary to know something about the asymptotic behavior of integrals of the form

$$
G(r, \theta, f)=\int_{\partial C} f(x) e^{i r(\theta, x)} d s_{x}
$$

where $d s_{x}=$ the surface area element, and $f(x)$ is a sufficiently smooth function on $\partial C$. The asymptotic behavior of $G(r, \theta, f)$ has been investigated, for general types of $\partial C$, by several authors ([4], [5], [6], [8], [9], [10]).

The result we will use in this paper is the following:

THEOREM A [9] (a more general $C^{\infty}$ version is given in [10]). If $\partial C$ is analytic, and if $C$ is convex (this is certainly unnecessary in $R^{2}$ ), then $|G(r, \theta, f)| \leqq \Phi(\theta) r^{-(n-1) / 2}$,

Received by the editors August 30, 1971.

AMS 1970 subject classifications. Primary 35P10, 42A62, 42A68, 42A92.

Key words and phrases. Asymptotic behavior of a Fourier transform, localization property for eigenfunction expansions. 
where $\Phi(\theta)$ is in $L^{p}\left(S^{n-1}\right)$, for some $p>2$. Moreover, the $L^{p}$ norm of $\Phi(\theta)$ depends only on bounds for $f(x)$ and a certain number, depending on $n$, of its derivatives in a neighborhood of $\partial C$. (In order to speak of the derivatives of $f(x)$, it suffices here, for example, to extend $f(x)$ homogeneously, say of weight 1 , and then take ordinary derivatives.)

It should be pointed out that there is an important question left unanswered by Theorem A. Namely, is the convexity requirement in dimensions greater than 2 superfluous or not? Insofar as I know, this is not known. If the requirement is unnecessary, one could extend localization theorems without qualification to all constant coefficient homogeneous elliptic operators on the torus.

I shall now describe the passage from $G(r, \theta, f)$ to $F_{\delta}(r, \theta)$. We begin with a lemma which occurs in [5], and whose proof is short enough to reproduce here.

Lemma (Hlawka).

$$
\int_{C}\left(1-(T(x))^{2}\right)^{\delta} e^{i r(\theta, x)} d v_{x}=2 \delta \int_{0}^{1}\left(1-t^{2}\right)^{\delta-1} t^{n+1} d t \int_{C} e^{i r t(\theta, x)} d v_{x}
$$

Proof.

$$
\begin{aligned}
\int_{C}\left(1-(T(x))^{2}\right)^{\delta} e^{i r(\theta, x)} d v_{x} & =\int_{C} e^{i r(\theta, x)} d v_{x} \int_{T(x)}^{1}-\left[\frac{d}{d t}\left(1-t^{2}\right)^{\delta}\right] d t \\
& =\int_{C} e^{i r(\theta, x)} d v_{x} \int_{T(x)}^{1} 2 \delta\left(1-t^{2}\right)^{\delta-1} t d t
\end{aligned}
$$

The last double integral is over a cone in $R^{n+1}$. Reversing the order of integration, the double integral becomes

$2 \delta \int_{0}^{1}\left(1-t^{2}\right)^{\delta-1} t d t \int_{t C} e^{i r(\theta, x)} d v_{x}=2 \delta \int_{0}^{1}\left(1-t^{2}\right)^{\delta-1} t^{n+1} d t \int_{C} e^{i r t(\theta, x)} d v_{x}$. Q.E.D.

By the divergence theorem, the double integral on the right in the statement of the lemma equals

$$
\frac{2 \delta}{i r} \int_{0}^{1}\left(1-t^{2}\right)^{\delta-1} t^{n} d t \int_{\partial C} e^{i t r(\theta, x)}(\theta, n(x)) d s_{x},
$$

where $n(x)$ is the outward normal to $\partial C$.

Now the major contribution to the integral in (1) comes from points near the "horizontal" part of $\partial C$, i.e. from points near the compact subset $M_{\theta}$ of $\partial C$ on which $n(x)$ is parallel to $\theta$. Indeed, if $f(x)$ is a $C^{\infty}$ function on $\partial C$ such that $f_{\theta}(x) \equiv 0$ in the complement of a small neighborhood of $M_{\theta}$, and $f_{\theta}(x) \equiv 1$ in a smaller neighborhood of $M_{\theta}$, we have

$$
\int_{0}^{1} \int_{\partial C}=\int_{0}^{1} \int_{\partial C} f_{\theta}+\int_{0}^{1} \int_{\partial C}\left(1-f_{\theta}\right)
$$


and we can choose $f_{\theta}(x)$ so that the contribution to (1) from the second double integral on the right is $O\left(r^{-n}\right)$, uniformly in $\theta[6, \mathrm{p} .767]$.

We are left with the integral

$$
\frac{2 \delta}{i r} \int_{0}^{1}\left(1-t^{2}\right)^{\delta-1} t^{n} d t \int_{\partial C} f_{\theta}(x) e^{i t r(\theta, x)}(\theta, n(x)) d s_{x} .
$$

LEMMA. $(\theta, x)$ is bounded away from zero on $M_{\theta}$, uniformly in $\theta$.

Proof. It suffices to show that $(\theta, x) \neq 0$ if $n(x)$ is parallel to $\theta$. This will be demonstrated if we can show that $x$ is never perpendicular to $n(x)$.

By Euler's differential equation for homogeneous functions,

$$
T(x)=x_{1} \frac{\partial T(x)}{\partial x_{1}}+\cdots+x_{n} \frac{\partial T(x)}{\partial x_{n}}
$$

and since $T(x) \neq 0$ unless $x=0$, and the right-hand side is proportional to $(x, n(x))$, the lemma is proved.

Now

$$
\begin{array}{rl}
\frac{2 \delta}{i r} \int_{0}^{1}\left(1-t^{2}\right)^{\delta-1} t^{n} & d t \int_{\partial C} f_{\theta}(x) e^{i t r(\theta, x)}(\theta, n(x)) d s_{x} \\
& =\frac{2 \delta}{i r} \int_{\partial C} f_{\theta}(x)(\theta, n(x)) d s_{x} \int_{0}^{1}\left(1-t^{2}\right)^{\delta-1} t^{n} e^{i t r(\theta, x)} d t .
\end{array}
$$

Assume for the moment that $\delta$ is an integer. Then if we integrate the inner integral on the right side by parts $\delta$ times, we find that

$$
\int_{0}^{1}\left(1-t^{2}\right)^{\delta-1} t^{n} e^{i t r(\theta, x)} d t=c e^{i r(\theta, x)}(i r(\theta, x))^{-\delta}-(i r(\theta, x))^{-\delta} \int_{0}^{1} h(t) e^{i t r(\theta, x)} d t,
$$

where $c$ and $h(t)$ are, respectively, a constant and a $C^{\infty}$ function, both of which are completely determined by $\delta$. Moreover, $h(t)$ has a zero of order $\geqq(n-1) / 2$ at $t=0$, since $0<\delta \leqq[(n-1) / 2]+1$. We conclude that

$$
\begin{aligned}
\frac{2 \delta}{i r} \int_{\partial C} f_{\theta}(x)(\theta, & n(x)) d s_{x} \int_{0}^{1}\left(1-t^{2}\right)^{\delta-1} t^{n} e^{i t r(\theta, x)} d t \\
= & \frac{2 \delta c}{(i r)^{\delta+1}} \int_{\partial C} f_{\theta}(x)(\theta, n(x))(\theta, x)^{-\delta} e^{i r(\theta, x)} d s_{x} \\
& -\frac{1}{(i r)^{\delta+1}} \int_{0}^{1} h(t) d t \int_{\partial C} f_{\theta}(x)(\theta, n(x))(\theta, x)^{-\delta} e^{i t r(\theta, x)} d s_{x} .
\end{aligned}
$$

Now inasmuch as $f_{\theta}(x)$ can be chosen so that $(\theta, x)$ is bounded away from zero on the support of $f_{\theta}(x)$, uniformly in $\theta$, and so that the derivatives of the homogeneous extension of $f_{\theta}(x)(\theta, x)^{-\delta}$ can be uniformly bounded in a neighborhood of $\partial C$, we can apply Theorem A to the integrals over $\partial C$ to obtain the following result: 
THEOREM B. If $\partial C$ is analytic, and if, for $n>2, C$ is convex, then $\left|F_{\delta}(r, \theta)\right|$ $\leqq \Phi(\theta) r^{-\delta-(n+1) / 2}$, where $\Phi(\theta)$ is in $L^{p}\left(S^{n-1}\right)$, for some $p>2$.

The theorem remains valid if $\delta$ is not an integer. In order to see this, we integrate the second integral on the right side of $(2)[\delta]$ times by parts to obtain

$$
\int_{0}^{1}\left(1-t^{2}\right)^{\delta-1} t^{n} e^{i t r(\theta, x)} d t=(\operatorname{ir}(\theta, x))^{-[\delta]} \int_{0}^{1} h(t) e^{i t r(\theta, x)} d t,
$$

where the function $h(t)$ is a sum of several terms, each of which, apart from a constant factor, is of the general form $\left(1-t^{2}\right)^{\alpha} t^{\beta}$, where $\alpha \geqq \delta-[\delta]-1$, and $\beta \geqq n-[\delta]$. Each of the corresponding integrals can be handled by the same standard method from the asymptotic theory of Fourier transforms [2, pp. 46-51], the only differences being in the number of integrations by parts that must be performed in order to either render the resulting expressions $O\left(r^{-\delta-(n-1) / 2}\right)$, or bring them into the standard form in which $-1<\alpha<0$. To take a specific example, the integral $\int_{0}^{1}\left(1-t^{2}\right)^{\delta-[\delta]-1} t^{n+[\delta]} e^{i t r(\theta, x)} d t$, which corresponds to one term of $h(t)$, is already in standard form, and requires no further integrations by parts. Formula 2.8(6) of [2] then tells us that this integral equals

$$
\begin{aligned}
c_{1} e^{i r(\theta, x)}(r(\theta, x))^{[\delta]-\delta} & +c_{2} e^{i r(\theta, x)}(r(\theta, x))^{[\delta]-\delta-1} \\
& +\cdots+c_{[(n-1) / 2]} e^{i r(\theta, x)}(r(\theta, x))^{[\delta]-\delta-[(n-1) / 2]}+R(r, \theta),
\end{aligned}
$$

where $R(r, \theta)=O\left((r(\theta, x))^{-[(n-1) / 2]-1}\right)$, and the constants $c_{1}, \ldots, c_{[(n-1) / 2]}$ do not depend on $\theta$. Integration over $\partial C$, bearing in mind Theorem $\mathrm{A}$, then yields the desired result.

We conclude with a brief description of the connection between Theorem B and localization theorems for elliptic differential operators on the torus.

Let $Q\left(\partial / \partial x_{i}\right)$ be a constant coefficient homogeneous elliptic operator of degree $\rho$ on the $n$-torus $T^{n}$, and let $T(x)=(Q(x))^{1 / \rho}$. The eigenfunctions of $Q\left(\partial / \partial x_{i}\right)$ are the functions $e^{i(N, x)}$, where $N$ is an integral lattice-point, and the corresponding eigenvalues are the numbers of the form $(i T(N))^{\rho}$. Now the eigenfunctions of $Q\left(\partial / \partial x_{i}\right)$ can be ordered in an intrinsic way by the corresponding eigenvalues. In particular, if

$$
c_{N}=(2 \pi)^{-n} \int_{T^{n}} f(x) e^{-i(N, x)} d v_{x}
$$

we define $S_{R}^{\delta}(x)(R>0, \delta \geqq 0)$ by setting

$$
S_{R}^{\delta}(x)=\sum_{|T(N)| \leqq R}\left(1-(T(N / R))^{2}\right)^{\delta} c_{N} e^{i(N, x)} .
$$

In the case in which $Q\left(\partial / \partial x_{i}\right)$ is the Laplacian, this reduces to spherical Riesz summation for Fourier series.

There are numerous questions which can be asked about the behavior of the Riesz sums $S_{R}^{\delta}(x)$, but we will only discuss one of them here, namely, is some reasonable version of Riemann's localization theorem true for this kind of sum- 
mation. I.e. if $f(x) \in L^{p}\left(T^{n}\right)$ for some $p \geqq 1$, and $f(x) \equiv 0$ in a neighborhood of the origin, is it true that for some $\delta$ independent of $f(x), S_{R}^{\delta}(0) \rightarrow 0$ ? It was shown by Riemann that the answer is yes for $T^{1}$, and that in that case one can take $\delta=0$, for any $p \geqq 1$. It was shown by Bochner that if $n>1$ and $Q\left(\partial / \partial x_{i}\right)$ is the Laplacian, then the answer is again yes for any $p \geqq 1$, provided that $\delta$ is greater than $(n-1) / 2$, the "critical exponent", but the theorem fails if $\delta=(n-1) / 2$ and $p=1$ [1]. It was also shown by Bochner in the same paper that if $p=2$ then localization holds at the critical exponent, and it was shown by Stein in [7] that localization holds at the critical exponent if $p>1$.

For illustrative purposes, we will give here a very simple example of a localization theorem for general operators, leaving aside the delicate question of validity at the critical exponent, and the discussion of general $L^{p}$ spaces. What we will show is that localization holds for any operator of the type under discussion, provided that $f(x) \in L^{2}\left(T^{n}\right)$ and $\delta>(n-1) / 2$, and provided that, in dimensions greater than 2 , the set $C=\{x \mid T(x) \leqq 1\}$ is convex. This possibly unnecessary convexity requirement is forced upon us by the present formulation of Theorem A, and it would, I think, be very interesting to know whether or not the convexity requirement in Theorem $A$ is superfluous.

The connection between Theorem $\mathrm{A}$ and localization is this: let $f^{*}(x)$ be the periodic extension of $f(x)$ to $R^{n}$, and for any $R>0$, let $F_{\delta}(R x)=F_{\delta}(R r, \theta)$, which was defined at the beginning of the paper. Then if $(n-1) / 2<\delta \leqq[(n-1) / 2]+1$, the function $f^{*}(x) F_{\delta}(R x)$ is, for any $R>0$, in $L^{1}\left(R^{n}\right)$, and

$$
S_{R}^{\delta}(0)=(R / 2 \pi)^{n} \int_{R^{n}} f^{*}(x) F_{\delta}(R x) d v_{x}
$$

(We will not discuss the validity of (3) when $\delta=(n-1) / 2$.)

In order to establish (3), note first of all that $F_{\delta}(x)$ is always $C^{\infty}$, and by Theorem $\mathrm{B}, F_{\delta}(x)$ is in $L^{1}\left(R^{n}\right)$. The Fourier inversion theorem thus applies to $F_{\delta}(x)$, and hence, for fixed $R$, to $(R / 2 \pi)^{n} F_{\delta}(R x)$. Supposing $f^{*}(x)$ to be of the form $\exp \left(-i\left(N_{0}, x\right)\right)$, we see that the right side of (3) equals $\left(1-\left(T\left(N_{0} / R\right)\right)^{2}\right)^{\delta}$ if $N_{0} \in R C$, and is zero otherwise. Formula (3) is therefore established for trigonometric monomials, and hence, by additivity, for trigonometric polynomials. If we can now show that

$$
f(x) \in L^{2}\left(T^{n}\right) \text { implies } f^{*}(x) F_{\delta}(R x) \in L^{1}\left(R^{n}\right),
$$

and that

$$
\int_{T^{n}}\left|f_{k}(x)\right|^{2} d v_{x} \rightarrow 0 \text { implies } \int_{R^{n}}\left|f_{k}^{*}(x) F_{\delta}(R x)\right| d v_{x} \rightarrow 0,
$$

formula (3) will follow by approximating $f(x)$ in $L^{2}\left(T^{n}\right)$ by trigonometric polynomials. In what follows, we may clearly assume that $R=1$. 
Now

$$
\int_{R^{n}}\left|f^{*}(x) F_{\delta}(x)\right| d v_{x}=\int_{r \leqq 1}+\sum_{k=1}^{\infty} \int_{k \leqq r \leqq k+1}
$$

But

$$
\int_{k \leqq r \leqq k+1}\left|f^{*}(x) F_{\delta}(x)\right| d v_{x} \leqq \int_{k \leqq r \leqq k+1}\left|f^{*}(x) \Phi(\theta)\right| r^{-\delta-(n+1) / 2},
$$

by Theorem B.

The last integral is dominated by $k^{-\delta-(n+1) / 2} \int_{k \leqq r \leqq k+1}\left|f^{*}(x) \Phi(\theta)\right| d v_{x}$. But

$$
\begin{aligned}
& \int_{k \leqq r \leqq k+1}|\Phi(\theta)|^{2} d v_{x} \leqq c k^{n-1}, \quad \text { for some } c>0, \text { and } \\
& \int_{k \leqq r \leqq k+1}\left|f^{*}(x)\right|^{2} d v_{x} \leqq c_{f} k^{n-1}, \quad \text { where } c_{f} \rightarrow 0 \text { as } \int_{T^{n}}|f(x)|^{2} d v_{x} \rightarrow 0 .
\end{aligned}
$$

Thus by the Schwarz inequality,

$$
\int_{k \leqq r \leqq k+1}\left|f^{*}(x) \Phi(\theta)\right| d v_{x} \leqq c_{f}^{\prime} k^{n-1},
$$

where $c_{f}^{\prime} \rightarrow 0$ as $\int_{T^{n}}|f(x)|^{2} d v_{x} \rightarrow 0$, and this establishes the desired conclusion.

We now pass from (3) to the localization theorem. Suppose $f^{*}(x) \equiv 0$ if $r<\varepsilon$. By (3),

$$
S_{R}^{\delta}(0)=(R / 2 \pi)^{n} \int_{R^{n}} f^{*}(x) F_{\delta}(R x) d v_{x}=(R / 2 \pi)^{n} \int_{r<\varepsilon}+(R / 2 \pi)^{n} \int_{r \geqq \varepsilon} .
$$

The first quantity is zero. By Theorem B, the second is dominated by

$$
R^{-\delta+(n-1) / 2} \int_{r \geqq \varepsilon}\left|f^{*}(x) \Phi(\theta)\right| r^{-\delta-(n+1) / 2} d v_{x} .
$$

By the argument used to establish (3), the integrand is in $L^{1}\left(R^{n}\right)$, and hence the last quantity tends to zero as $R \rightarrow \infty$, which establishes the localization theorem if $(n-1) / 2<\delta \leqq[(n-1) / 2]+1$. Theorem 16 of [3] then implies that the theorem is true for all $\delta>(n-1) / 2$.

\section{REFERENCES}

1. S. Bochner, Summation of multiple Fourier series by spherical means, Trans. Amer. Math Soc. 40 (1936), 175-207.

2. A. Erdélyi, Asymptotic expansions, Dover, New York, 1956. MR 17, 1202.

3. G. H. Hardy and Marcel Riesz, The general theory of Dirichlet's series, Cambridge Univ. Press, Cambridge, 1952.

4. C. S. Herz, Fourier transforms related to convex sets, Ann. of Math. (2) 75 (1962), 81-92. MR $26 \# 545$.

5. E. Hlawka, Uber Integrale auf konvexen Körpern. I, Monatsh. Math. 54 (1950), 1-36. MR 12, 197. 
6. Walter Littman, Fourier transforms of surface-carried measures and differentiability of surface averages, Bull. Amer. Math. Soc. 69 (1963), 766-770. MR 27 \#5086.

7. E. M. Stein, Localization and summability of multiple Fourier series, Acta Math. 100 (1958), 93-147. MR 21 \#4331.

8. Burton Randol, On the Fourier transform of the indicator function of a planar set, Trans. Amer. Math. Soc. 139 (1969), 271-278. MR 40 \#4678a.

9. - On the asymptotic behavior of the Fourier transform of the indicator function of a convex set, Trans. Amer. Math. Soc. 139 (1969), 279-285. MR 40 \#4678b.

10. Ingvar Svensson, Estimates for the Fourier transform of the characteristic function of a convex set, Ark. Mat. 9 (1971), 11-22.

Department of Mathematics, Graduate Center, City University of New York, New York, New York 10036 